\title{
Announcement
}

\section{Myotubular Trust - 2019 Call for Projects (Open to International Applications)}

\section{Background}

The Myotubular Trust (UK Registered Charity No: 1137177) was set up in 2006 to raise money for research to find a cure and / or treatment for any of the genetic forms of centronuclear and myotubular myopathy.

There are a number of genetically distinct forms of centronuclear myopathy. The commonest is x-linked, usually called myotubular myopathy, affecting in the vast majority of cases, only boys. It is also usually the most severe, presenting in the new-born period and there are associated breathing and swallowing difficulties in addition to the general muscle weakness. The other forms are either dominant or recessive in inheritance, are usually, but not always, milder, and vary widely.

\section{9 call for projects}

Myotubular Trust is holding a 2019 call for research grants. We will require completed applications by 1700 hours GMT Friday 15th March 2019. We anticipate making awards in late June / early July.

We are looking to fund further projects that will help find a cure and / or a treatment for any form of centronuclear and myotubular myopathy (congenital $\mathrm{X}$-linked recessive; congenital autosomal recessive; autosomal dominant), focusing on research that would not generally be funded by public or industrial funding sources. This call will be open to research bodies internationally.
We will be looking for the following types of application:

1. A project grant applied for by a Principal Investigator to fund a project for 2-3 years duration to be carried out by a Post-Doctoral researcher, or PHD student

2. A Myotubular Trust fellowship - basic science (3-4 years duration), where the scientist has identified a group that he or she wants to work with. Award is made to a named individual.

In particular, we would like to encourage the application of new technologies to research into centronuclear and myotubular myopathy; interventional trials; and those which may involve collaboration between different medical disciplines and / or different research institutions. We are also willing to consider applications which involve joint funding with other organisations.

Myotubular Trust's Scientific Advisory Board (SAB) is chaired by Professor Francesco Muntoni of The Institute of Child Health, University College London. The SAB makes recommendations to the Myotubular Trust Trustees on which projects to fund, based on scientific assessment and peer review.

\section{Further information}

Further guidance and an application form can be found on the website - https://myotubulartrust.org/ research/grants-process/ 
If you wish to learn more about the Myotubular Trust, please see our website http://www.myotubulartrust. org or email research@myotubulartrust.org
If you wish to learn more about the Myotubular Trust, please see our website http://www.myotubulartrust. org or email research@myotubulartrust.org

\section{Anne Lennox - Parent Trustee}

Myotubular Trust, 1137177

http://www.myotubulartrust.org

Founding Patron: Professor Victor Dubowitz, Emeritus Professor of Paediatrics, Imperial College London; President of World Muscle Society.

Scientific Advisor: Professor Francesco Muntoni, Professor of Paediatric Neurology, University College London.

$100 \%$ of our supporters donations goes directly to pay for research and nothing else. Our core and administration costs are kept to an absolute minimum and are paid for by specific private donation.

Registered charity no 1137177. Disclaimer: The Myotubular Trust is committed to respecting the privacy rights of its donors, supporters and affiliates, therefore the contents of this email are confidential and intended solely for the use of the person or organisation to whom it is addressed. If you are not the intended recipient, you should not copy, distribute or take any action with regards this e-mail. Any opinions expressed in this e-mail are those of the author and do not necessarily reflect the opinions of the Myotubular Trust and its affiliates. Although the Myotubular Trust endeavours to ensure e-mails and attachments are free from viruses prior to being sent, the charity will not be liable for any losses as a result of any viruses being passed on. 